\title{
FLIPPED CLASSROOM, LCA Y MATERIALES DE CONSTRUCCIÓN: UNA EXPERIENCIA DIDÁCTICA PARA UNA ACTIVIDAD DE APRENDIZAJE COOPERATIVA Y ACTIVA
}

FLIPPED CLASSROOM, LCA AND CONSTRUCTION MATERIALS: A DIDACTIC EXPERIENCE FOR A COOPERATIVE AND ACTIVE LEARNING ACTIVITY

\author{
S. Rizzo ${ }^{1}$, F. Capellaro ${ }^{2}$, J.R. Ruiz-Checa ${ }^{3}$, V. Cristini ${ }^{3}$ \\ ${ }^{1}$ University of Bologna, Italia \\ ${ }^{2}$ ENEA (Italian National Agency for New Technologies, Energy and Sustainable \\ Economic Development) Roma, Italia \\ ${ }^{3}$ Universitat Politècnica de València, España \\ vacri@cpa.upv.es
}

\begin{abstract}
RESUMEN
El texto presenta una experiencia didáctica interdisciplinar que se está llevando a cabo en el Campus Terracini de la Università di Bologna (por miembros académicos de la Universidad de Bolonia investigadores del centro ENEA y de la Universitat Politècnica de València). La actividad se centra en la realización de un pequeño pabellón, sede del Trasition Team, un grupo experimental, involucrados en temáticas ambientales y sostenibles, internas y externas a la dinámica académica del Campus. Para ello, se ha estructurado una experiencia didáctica constructiva, centrada en este caso, en el empleo de materiales locales, que cumplen con requisitos determinados, prestaciones energéticas e impacto sostenible. Gracias a esta experiencia participativa de autoconstrucción, basada en la aplicación, análisis y definición del LCA de algunos materiales constructivos se está experimentando una propuesta didáctica alternativa, basada en el concepto de clase inversa o flipped classroom, crucial para futuros profesionales vinculados directa e indirectamente al mundo de la construcción.

Palabras clave: aprendizaje experimental, LCA, materiales, transition
\end{abstract}

\begin{abstract}
The paper outlines a didactic project undertaken at Campus Terracini (University of Bolonia Italy) thanks to local staff members and with the support of researchers from ENEA agency, Italy and professors of Polytechnic University of Valencia, Spain. The activity tries to To make sustainability transitions happen, considering that it is crucial not only to have a strategic planning processes committed by the top management, but also to encourage community engagement, approaching and promoting a bottom-up process. For this reason the project is concerning the layout of a small pavilion, head quarter of Transition Team (a multifunction group involved in sustainability plan of Unibo). To meet environmental performances, the space is planned with the use of appropriate building technologies, employing low impact and local materials. In addition, the space is realized in auto-construction, in order to strengthen the involvement of final users, the students. This paper will show an evaluation of appropriate building technologies with an LCA approach, combined with flipped classroom didactic methodology.
\end{abstract}

Key words: experimental learnig, LCA, sustainability transition and raw materials 


\section{INTRODUCCIÓN}

Este trabajo presenta una experiencia docente donde se emplea la clase invertida, flipped classroom (Lage, 2000; Foertsch, 2002) como proceso de experimentación docente en el campo de la sostenibilidad y donde confluyen teorías y prácticas de enseñanza, todas ellas relacionadas con este tema. El proyecto se está llevando a cabo en la Universidad de Bolonia, Italia, Campus Terracini, con el apoyo del centro ENEA (Italian National Agency for New Technologies, Energy and Sustainable Economic Development) y con la asesoría de miembros de la Universitat Politècnica de València (gracias al proyecto Pioneers into Practice Program- Climate KIC-UE).

Desde 2013, la Universidad de Bolonia ha desarrollado un Plan de la Sostenibilidad que ofrece un marco especial donde se pueden desarrollar iniciativas ecológicas tanto en el conjunto de la Universidad como en determinados campus de la misma (Bonoli, Cappellaro, 2013). En concreto, en el Campus de Terracini, se ha creado un grupo multidisciplinar, compuesto por los diferentes sectores de la comunidad universitaria (profesores, estudiantes, PAS) gracias al DICAM (Department of Civil, Chemical, Environmental and Materials Engineering de la Universidad de Bolonia Italia).

Este grupo se denomina The Terracini Transition Team y está compuesto por profesores, estudiantes de doctorado, investigadores y estudiantes, todos ellos adscritos al Campus de Terracini. La mayor parte de las acciones tienen como principal objetivo mejorar la educación/aprendizaje entre los estudiantes de Ingeniería a través de prácticas de laboratorio junto con acciones relativas a investigación y pedagogía. Justo en este marco se perfila el proyecto indicado a continuación, cuyo objetivo es la autoconstrucción de un pabellón sede del grupo, llevada a cabo por mismos miembros de la comunidad universitaria. Todo ello con la finalidad de contribuir en la transformación del campus universitario como living-lab (laboratorio viviente) de la sostenibilidad (UNEP, 2013).

\section{EL CONCEPTO DE CAMPUS SOSTENIBLE}

Aunque un gran número de iniciativas internacionales están promoviendo la creación de campus sostenibles (Calder, Clugston, 2003; Wright, 2004) existen aún aspectos que requieren de mayor compromiso académico para que varios paradigmas lleguen algún día a ser parte integral del sistema universitario (Lozano, 2013, Bekessy et al., 2007, Thaman, 2002). Existe por tanto, la necesidad de superar el actual enfoque fragmentado, para alcanzar una visión global basada en un desarrollo sostenible hólista (Cortese, 2003; Burke, 2000). Por ello, la adopción de sistemas integrales de análisis y la creación de procesos proactivos contribuyen a la integración de teorías y prácticas sostenibles dentro de los campus, en concreto, referidas a la adopción de nuevos paradigmas educativos, (Sharp, 2002; Koester, 2006).

De acuerdo con UNEP 2013 (United Nations Environment Programm, 2013), la combinación de un fuerte compromiso de gestión, por parte del profesorado, junto con una alta participación de los estudiantes, garantiza el mejor escenario posible para engendrar una transformación exitosa, así como una perspectiva a largo plazo en la aplicación de programas de sostenibilidad dentro los campus universitarios. En este marco, un papel crucial que pueden jugar los estudiantes, 
es formar parte de estas iniciativas, pudiendo ser creadores de conocimiento, agentes o responsables del cambio (Müller-Christ et al., 2014).

De acuerdo con Kolb (1984), "el aprendizaje es el proceso por el cual el conocimiento se crea a través de la transformación de la experiencia". Por lo tanto, el proceso de aprendizaje puede llegar a ser un momento experimental de formación gracias al empleo de problemas de la vida cotidiana, como "contextopretexto" donde los alumnos aprenden de manera crítica y adquieran habilidades para resolver problemas (Hansman, 2001; Bould and Felletti, 1991).
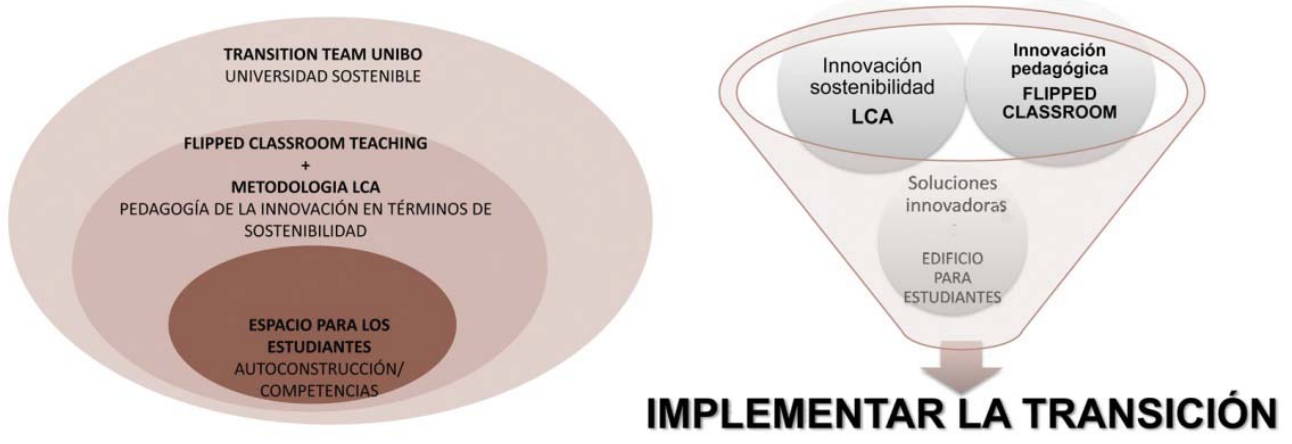

Fig.1. Esquema del modelo didáctico finalizado a implementar una experiencia de transición (autores)

Con estos antecedentes, este texto presenta un innovador y fascinante idea a partir de la cual se podría plantear una considerable mejora en la sostenibilidad llevada a cabo en el Campus de Terracini de la Universidad de Bolonia, a partir de la materialización de un espacio específico destinado a los alumnos. Este, puede convertirse además en un espacio donde sea posible mejorar un enfoque participativo y presentar una oportunidad interesante para poner en práctica conceptos de sostenibilidad.

\section{EL PROYECTO DIDÁCTICO}

El proyecto propone que los estudiantes participantes aborden todas las fases de ejecución de un espacio construido mediante criterios de sostenibilidad aplicada a propuestas proyectuales y materiales de construcción. Desde la fase de diseño del espacio y su implantación hasta la selección de tecnologías constructivas y/o materiales de construcción. Para ello el método se basa en empleo del standard LCA (Life Cycle Assessment), tomando como consigna que los recursos empleen la menor cantidad de energía (materiales locales, bajo impacto ambiental). Por ello, en la fase de ejecución se propone que los propios estudiantes sean los responsables de su ejecución (autoconstrucción). En este sentido, involucrar a los futuros usuarios (los estudiantes) supone una de las grandes fortalezas del proyecto. Además, la fase real de utilización del espacio se ha planteado para garantizar que la construcción ofrezca un alto estándar de eficiencia energética y generación mínima tanto de residuos como de consumo de materiales.

En esencia, el objetivo primordial del presente trabajo se dirige a demostrar como la adopción del concepto de sostenibilidad como sistema integral de actuación puede contribuir a convertir a dicho paradigma en parte integrante del mundo universitario. Definitivamente, la transformación del campus universitario en un lugar altamente sostenible puede ayudar a la sociedad, a largo plazo, a aumentar la concienciación en aspectos ambientales y referidos a la sostenibilidad. 


\subsection{El Recurso: Flipped Classroom}

Para el desarrollo de la presente experiencia en el Campus de Terracini (Universidad de Bolonia, Italia) se propone como base de trabajo la metodología y resultados empleados en el proyecto de investigación sobre sostenibilidad, VERSUS "Lesson from Vernacular Heritage to Sustainable Architecture" (Correia et al., 2012, 2014a). De esta manera, los estudiantes desarrollan un proyecto básico del pabellón, evaluado según un checklist recogido en el Proyecto VERSUS. (Correia et al., 2014b)
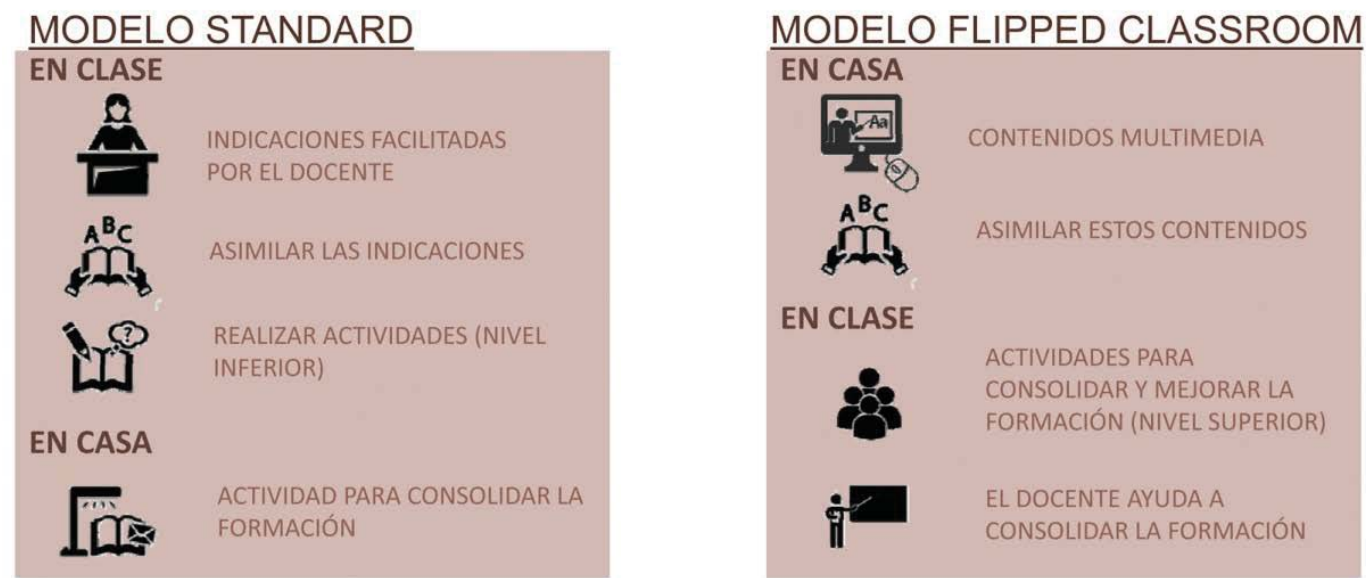

Fig.2. Esquema del modelo de docencia "standard" comparado con el modelo flipped classrom (autores)

Durante cada clase, se lleva a cabo un análisis riguroso y detallado desde diferentes enfoques. Además, en cada sesión se trabaja sobre cada uno de los 15 principios sostenibles que se proponen en el proyecto VERSUS (Mileto et al., 2015a, 2015b). Estos principios están agrupados en tres ámbitos diferentes, por tanto cada estudiante debe ser consciente en todo momento de qué ámbito o en qué escenario se está actuando. Así, estos quince principios quedan agrupados de la siguiente manera:

-Ámbito medioambiental (5 principios): Respetar la naturaleza, implantarse adecuadamente, disminuir la contaminación y los desechos, preservar la salud, minimizar los efectos de los riesgos naturales.

-Ámbito Socio-Cultural (5 principios): proteger el paisaje cultural, transmitir las culturas constructivas, suscitar la creatividad, reconocer los valores inmateriales, favorecer la cohesión social.

-Ámbito Socio-Económico (5 principios): fomentar la autonomía, promover la actividad local, optimizar los esfuerzos de construcción, prolongar la vida útil de los edificios, ahorrar recursos. 


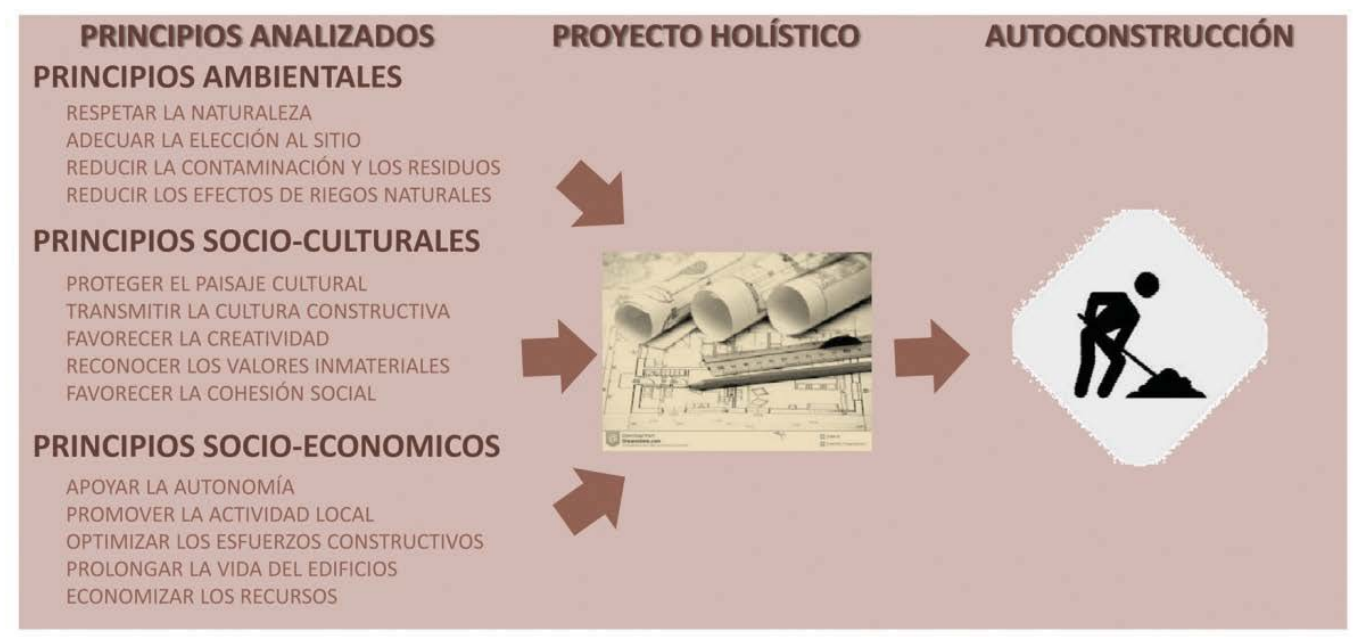

Fig.3. Resumen de las fases de análisis basadas en los principios identificados por los estudiantes (autores)

Previamente a la actividad en clase, además, los estudiantes tienen a su disposición diferentes recursos y contenidos (videos, enlaces, ejemplos, bibliografía, aplicaciones) basados en los principios que se tratarán en la sesión de aula. Una vez se inicia la clase, el modelo docente sigue el siguiente esquema:

-Evaluación o sondeo inicial sobre los contenidos y recursos que se han suministrado previamente a los alumnos. De esta manera, el profesor puede evaluar el nivel general alcanzado por los estudiantes y su grado de compromiso o deficiencia (5min.).

-Sesión abierta de coloquio sobre los resultados, de esta manera los propios alumnos son conscientes de sus deficiencias en el proceso de aprendizaje.

-Puesta en común de las cuestiones que han surgido. De este modo, dichos asuntos, analizado de modo participativo ayudan al resto de la clase.

-Progresión en el estudio. Una vez los estudiantes han trabajado en un nivel inferior (entender, recordar/Taxonomía de Bloom), el siguiente paso se basa en el avance del estudio (evaluar y analizar/Taxonomía de Bloom) calibrando los principios relativos a la sostenibilidad que previamente se han mencionado.

-Puesta en común y discusión de los resultados de las diferentes tareas y selección de los aspectos más interesantes de los principios analizados.

-Fase final de consolidación. El profesor procede a interactuar con los alumnos para que se involucren en el proceso pedagógico a través de actividades basadas en los 15 principios tratados.

\subsection{La herramienta: el estándar LCA}

Después de las sesiones de clase donde se han diseñado, creado y evaluado las propuestas proyectuales, se propone un momento de puesta a punto de la práctica. Así es como se procede a iniciar la construcción del futuro espacio por los propios alumnos del campus. Por tanto, la elección de los mejores materiales y técnicas más sostenibles se basan en primera instancia en los principios rectores del proyecto VERSUS. Ahora bien, esta primera elección de sistemas y materiales viene confirmada por la evaluación llevada a cabo a través del standard LCA (Life Cycle Assessment).

En este contexto, tal y como confirman los estudios realizados por los participantes del proyecto, muros construidos con balas de paja y revestido de 
morteros de tierra constituyen la solución constructiva con mejores prestaciones, considerando los criterios y parámetros de sostenibilidad adoptados. Estas soluciones se ajustan desde diferentes vertientes (social, cultural o económica) a la realidad local del Campus de Terracini, logrando de esta manera una experiencia basada en el concepto de abastecimiento de materiales de construcción a "KM 0", vinculada a los recursos autóctonos de la Llanura Padana.

Con el objetivo de evaluar la sostenibilidad de los materiales elegidos (balas de paja y mortero de tierra), se ha recurrido al stándar LCA (Life Cycle Assessment -ISO 14040, ISO 14044). Uno de los pasos más interesantes a destacar en el proceso ha sido el enfoque basado en el concepto "desde la cuna hasta la tumba" (from cradle to grave) de los acabados del pabellón a ejecutar, fase de estudio donde concretamente los autores han estado más directamente involucrado, a lo largo de diferentes fases de análisis prestacional.

\section{LCA TECNOLOGÍA ADECUADA PARA LA CONSTRUCCIÓN DIAGRAMA DE FLUJO DEL PROCESO ESTUDIADO}

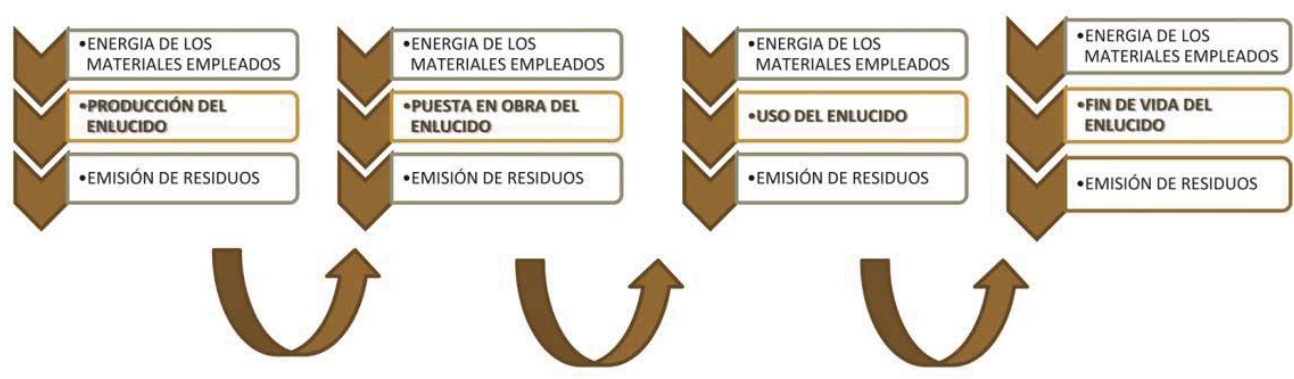

Fig.4. Esquema de la aplicación del stándard LCA en el marco del proyecto propuesto(autores)

En este marco, se parametriza con detalle una unidad de referencia $\left(1 \mathrm{~m}^{2)}\right.$ de mortero, suponiendo un ciclo de vida útil de 100 años (fase de producción del mortero, fase de aplicación, fase de uso, fase fin de vida /EOL/ End Of Life).

A partir de fases de análisis, inspección de otras construcciones y de entrevistas a proyectistas y constructores ha sido posible recopilar una gran cantidad de datos necesarios para llevar a cabo un análisis de LCA riguroso de paja y mortero de tierra, que serán los materiales cruciales para la puesta a punto de la programación de la obra.

\section{CONCLUSIONES}

El proyecto, basado en la aplicación de principios sostenibles a una experiencia didáctica y constructiva real, propone que los estudiantes participantes aborden todas las fases de un proceso constructivo vinculados al proyecto de un pequeño pabellón. Desde la fase inicial (introducción al proyecto), mediante el método flipped classroom, o el diseño del espacio y su implantación...hasta la fase de selección de tecnologías constructivas con el empleo del estándar LCA (Life Cycle Assessment), los alumnos protagonizan fases de aprendizaje activo. Tomando como consigna que los materiales constructivos empleen la menor cantidad de energía (materiales locales, bajo impacto ambiental, etc.) los propios estudiantes se responsabilizan e involucran plenamente en la ejecución (autoconstrucción) del espacio que van a emplear. 
Durante el desarrollo de la práctica, el papel de los alumnos es activo en el proceso de aprendizaje/enseñanza, siendo el profesor un gestor y un apoyo de este proceso. Lo novedoso de dicha experiencia radica en que, al contrario de lo que ocurre en otras experiencias similares, la evidencia sigue evaluándose y experimentándose por los propios alumnos, son usuarios. Este principio es fundamental en las enseñanzas de disciplinas vinculadas al mundo de la construcción donde los criterios como mantenimiento, durabilidad o confort son difícilmente evaluables. Learning by doing, pero un paso más allá, Learning by living.

\section{BIBLIOGRAFÍA}

Bekessy, S., K. Samson, and R.E. Clarkson (2007) "The failure of non-binding declarations to achieve university sustainability: A need for accountability". International Journal of Sustainability in Higher Education, 2007. 8 (3): p. 301316

Bonoli A., Cappellaro F., (2013) "Initiatives to start with implementing sustainability process into University of Bologna", in CUCSTorino2013 Congress of the Italian University Network for Development Cooperation (CUCS), Turin, 1921 September 2013

Bonoli A., Rizzo S., Chiavetta C., (2014) Straw as construction raw material for sustainable buildings, VerSusValencia2014 Vernacular Heritage and Earthen Architecture: Contributions for Sustainable Development, p. 143-146

Bould, D. and Felletti G. (1991) eds. The Challenge of Problem-Based Learning. St. Martin's Press: New York

Burke, J. (2000) The Knowledge Web, first ed. Touchstone, New York

Calder, W., Clugston, R.M., (2003) "International efforts to promote higher education for sustainable development" in Planning for Higher Education 31, 3044.

Cappellaro, F., Bonoli, A. (2014) "University in Transition. How to transform campus into living laboratory of sustainability" in Proceedings of 5th International Conference on Sustainability Transitions, Utrecht, 27-29 August

Correia M., Carlos G., Rocha S., (2014), Vernacular Heritage and Earthen Architecture: Contributions for Sustainable Development, Eds. Correia, Carlos \& Rocha, Balkema Publisher, London

Correia M., Mileto C., Vegas F., (2012), PROJECT VERSUS Lesson from Vernacular Heritage to Sustainable Architecture, Union Culture Programme, European Pro $\mathrm{n}^{\circ}$ 2012-2792/001-001 CU7 COOPP7, On line at: http://www.esg.pt/versus/

Cortese, A.D. (2003) "The critical role of higher education in creating a sustainable future" in Planning for Higher Education 31 (3), 15-22

Foertsch J., Moses G., Strikwerda J., Litzkow M., (2002), "Reversing the Lecture/Homework Paradigm Using eTEACH Web-based Streaming Video Software" in Journal of Engineering Education, 91, 267-274

Fugler, D. (2000) "Moisture properties of plaster and stucco for straw bale buildings", in CMHC Canada Mortage and Housing Corporation, Research Highlight Technical Series, 132 (2000)

Fugler, D. (2002) "Energy use in straw bale houses", in CMHC Canada Mortage and Housing Corporation, Research Highlight Tecnical Series, 115 (2002)

Hansman, C.A. (2001) Context-based Learning, in New Directions for Adult and Continuing Education, S. Merriam, Editor. Jossey-Bass: San Francisco. p. 43-52 
ISO 14040:2006, Environmental management-life cycle assessment / principles and frame work

ISO 14044:2006, Environmental management-life cycle assessment I requirements and guideline

Koester, R.J. Eflin, J., and Vann, J. (2006) "Greening of the campus: a wholesystems approach" in Journal of Cleaner Production, 14: p. 769-779.

Kolb, D. (1984). Experiential Learning, New Jersey: Prentice Hall.

Lage, M.J., Platt, G. J., Treglia, M., (2000). "Inverting the classroom: a gateway to creating an inclusive learning environment", in Journal of Economic Education, 31(1), 30-43

Lozano, R., Lukman, R., Lozano, F.J., Huisingh, D., Lambrechts, W. (2013) "Declarations for sustainability in higher education: becoming better leaders, through addressing the university system" in Journal of Cleaner Production, 48 (2013) 10-19

Mileto C., Vegas F., García-Soriano, L., Cristini V., (2015), Earthen Architecture: Past, Present and Future, Eds. Mileto, Vegas, García Soriano \& Cristini, Balkema Publisher, London

Mileto C., Vegas F., García-Soriano L., Cristini V., (2015), Vernacular Architecture: Towards a Sustainable Future, Eds. Mileto, Vegas, García Soriano \& Cristini, Balkema Publisher, London

Müller-Christ G., Sterling S., van Dam-Mieras R., Adomßent M., Fischer D, Rieckmann M. (2014) "The role of campus, curriculum, and community in higher education for sustainable development: a conference report" in Journal of Cleaner Production 62 (2014) 134-137

Sharp, L. (2002)"Green campuses: the road from little victories to systemic transformation" in International Journal of Sustainability in Higher Education, 2002. 3(2): p. 128-145

Thaman, K. (2002) "Shifting sights: The cultural challenge of sustainability" in International Journal of Sustainability in Higher Education, 2002. 3(3): p. 233-242

UNEP, United Nations Environment Programme (2013) Greening Universities

Toolkit, http://www.unep.org/training/docs/Greening_University_Toolkit.pdf

Wright, T. (2004) "The evolution of sustainability declarations in higher education". In: Corcoran, P.B., Wals, A.E.J. (Eds.), in Higher Education and the Challenge of Sustainability: Problematics, Promise, and Practice. Kluwer Academic Publishers, Dordrecth, The Netherlands 